\title{
Ökonomik: Eine Moraltheorie mit religiösen Wurzeln*
}

\author{
BENJAMIN M. FRIEDMAN ${ }^{* *}$
}

Dass die Ökonomik eine Moraltheorie ist, ist für Wirtschaftsethiker keine Überraschung. Dieser Beitrag zeigt aber auf, in welcher Weise religiöse Wurzeln die Ökonomik bei Ihrer Entstehung prägten und inwiefern der Wandel religiöser Ideen auch den Wandel in Bezug auf die Akzeptanz einer bestimmten Form von Ökonomik beeinflusst hat. Dabei wird auf vier entscheidende religiöse Vorstellungen verwiesen, die sich einerseits im Zuge des Niedergangs des orthodoxen Calvinismus entwickelten und andererseits im Rahmen der Debatte um Prä- und Postmillenaristen thematisiert wurden. Auch ohne den einschlägigen Autoren, wie Adam Smith, bestimmte religiöse Überzeugungen zuzusprechen, scheint das religiös geprägte mentalitäts- und ideengeschichtliche Umfeld maßgeblich die Ökonomik als Moraltheorie beeinflusst zu haben.

Schlagwörter: Theoriegeschichte, Ökonomie, Religion, Adam Smith

\section{Economics: A Moral Inquiry with Religious Origins}

It may not come as a surprise for Business Ethicists that economics is a moral theory. But this article shows in what way religious roots have shaped economics in its emergence and how changes of religious ideas have also influenced the change with regard to the acceptance of a certain form of economics. Hereby, it will be referred to four crucial religious concepts, which on the one hand have developed in the course of the decline of the orthodox Calvinism and on the other hand have been made subject of discussion in the course of the debate about Pre-and Postmillenarists. Without attributing the relevant authors, like Adam Smith, certain religious convictions, the bighly religious influenced environment of ideas and attitudes seems to have influenced economics as moral theory in a significant way.

Keywords: History of Economic Thought, Economics, Religion, Adam Smith

Ungeachtet ihrer festen Fundierung als empirisch gestützte Disziplin war die Ökonomik ursprünglich eine ethische Wissenschaft. Adam Smith war Professor für Moralphilosophie. Das spiegelt sich nicht nur in seiner früheren Schrift, der Theorie der ethischen Gefüble, sondern auch im Woblstand der Nationen wider. Beide Bücher enthalten zahlreiche Analysen individueller Motive und psychologischer Zustände. Sie analysieren zudem die Art und Weise, in der „ökonomische“ Aktivitäten - wie wir sie heute nennen, und die ja grundsätzlich immer in gesellschaftlichen Bezügen stattfinden - die

\footnotetext{
* Ursprünglich veröffentlicht als: Economics: A Moral Inquiry with Religious Origins, in: American Economic Review, Vol. 101/No. 3 (2011), pp. 166-170; mit freundlicher Genehmigung des Autors übernommen und ins Deutsche übersetzt von Philip Krug.

** Benjamin M. Friedman, Harvard University, Department of Economics, Littauer Center 127, US-02138 Cambridge, MA, Tel.: +1-(0)617-495-42246, E-Mail: bfriedman@harvard.edu, Forschungsschwerpunkte: Geschichte ökonomischen Denkens, intellektuelle Ursprünge der Ökonomik, Ökonomik und Religion, Ökonomik und Gesellschaft.
} 
Menschen in die Lage versetzen, ein befriedigendes Leben zu führen - oder eben nicht. Selbst die Arbeitsteilung, die Smith bereits im ersten Satz des Woblstands der Nationen als den Schlüssel für Produktivitätssteigerungen so nachhaltig befürwortete, unterliegt ausdrücklichen moralischen Bedenken - weil sie die Individuen nicht nur „differenzierte[r] Empfindungen, wie Selbstlosigkeit, Großmut oder Güte“ beraubt, sondern ihnen auch die Urteilsfähigkeit gegenüber den „Dingen [...] des täglichen Lebens“ oder den „wichtigen und weitreichenden Interessen“ des Landes nimmt. Sein größtes Anliegen ist es, das Wohlergehen von dem zu fördern, was Smith „das großartige Gefüge des Volkes“ nennt. Materieller Wohlstand hat einen eigenständigen Wert, aber - und das ist noch bedeutender - er ist eine notwendige Voraussetzung für das individuelle Glück und den gesellschaftlichen Fortschritt: „Und ganz sicher kann keine Nation blühen und gedeihen, dessen Bevölkerung weithin in Armut und Elend lebt." 1

Als würden Smiths Bedenken gegenüber der Arbeitsteilung bestätigt, wird der moralische Aspekt unserer Disziplin in der heute immer enger und spezialisierter werdenden Form der ökonomischen Analyse häufig ignoriert und manchmal sogar bewusst verdrängt. Aber dieser Aspekt war von Anfang an vorhanden und er wird auch weitgehend anerkannt. Was aber in der Regel nicht verstanden wird - und was tatsächlich auch den gegenwärtigen Interpretationen des Ursprungs der Ökonomik als eigenständige intellektuelle Disziplin widerspricht - ist der Einfluss, der nicht von ethischen, sondern von im klassischen Sinne religiösen Ideen auf die Arbeit von Smith und anderen frühen „Ökonomen“ rührt.

\section{Religiöse Einflüsse auf ökonomisches Denken?}

Heute wird allgemein angenommen, dass die Ökonomik aus der europäischen Aufklärung des 18. Jahrhunderts im Zuge der allgemeinen Bewegung in Richtung einer säkularen Moderne entstanden ist, und zwar im Sinne einer historischen Wendung des Denkens weg von einem Gott-zentrierten Universum hin zu dem, was wir gemeinhin Humanismus nennen. Im Gegensatz dazu schlage ich vor, dass die überaus wichtige Veränderung im Denken, die wir aus gutem Grund mit Adam Smith, seinen Zeitgenossen und Schülern verbinden - also der entscheidende Wandel, der uns die Ökonomie, wie wir sie kennen, brachte - stark von damaligen Veränderungen der Glaubensüberzeugung in der englischsprachigen protestantischen Welt, in welcher sie lebten, beeinflusst wurde. Ferner haben diese anfänglichen Einflüsse religiösen Denkens nicht nur die anschließende Verbreitung der Ansichten Smiths vor allem in Amerika begünstigt, sondern sie beeinflussten auch den Verlauf ihrer Rezeption. Im Endergebnis führte dies zu einem vielfältigen Wiederhall zwischen ökonomischem und religiösem Denken, der heute nach wie vor unsere öffentliche Diskussion ökonomischer Themen und unsere öffentliche Debatte über Wirtschaftspolitik beeinflusst.

Die Idee eines religiösen Einflusses auf Adam Smiths Ansichten oder auf jene seiner Zeitgenossen und Schüler wird wahrscheinlich vielen Lesern der American Economic Review unplausibel erscheinen. Soweit wir das aus den verfügbaren biografischen Quellen beurteilen können, war Smith, wie viele andere Persönlichkeiten der Aufklärung, höchstens das, was viele amerikanische Gelehrte dieser Zeit als Jefferson'schen Deist

1 Wörtliche Zitate sind entnommen aus Adam Smith (1776), übers. durch H. C. Recktenwald. 
bezeichnen. Es gibt wenige Anhaltspunkte dafür, dass sich Smith in religiösen Fragen aktiv beteiligt hätte, und noch weniger dafür, dass er sich für Religion begeistert hätte. ${ }^{2}$ Aber Smith und seine Zeitgenossen lebten in einer Zeit, in der die Religion sowohl weiter verbreitet als auch gesellschaftlich wesentlich bedeutsamer war, als wir das heute in der westlichen Welt kennen. In Großbritannien zur Zeit Smiths waren Religion und Politik deckungsgleich. Die Moderates und die Evangelicals in der Church of Scotland haben selbstverständlich religiöse Fragen thematisiert. Aber sie diskutierten auch über politische Angelegenheiten: Fragen zur Freiheit wie dem zulässigen Grad an Toleranz, die Frage der Kirchenautorität und damit auch des politischen Einflusses der Kirche sowie Angelegenheiten des Patronats bei der Vergabe von Pfründen, Ehrentiteln und Ämtern. Auf eine wesentlich dramatischere Art und Weise bezogen sich im Jahrhundert zuvor die gesellschaftlichen Kontroversen des Englischen Bürgerkriegs, des Puritanischen Commonwealth unter Cromwell, der Stuart-Restauration und der Glorreichen Revolution von 1688 auf religiöse Auseinandersetzungen. Aber auch Smith war Zeuge dieses Phänomens; er war 22 Jahre alt, als 1745 der Aufstand der Jakobiten die gleiche tödliche Mischung aus Religion und Politik nach Schottland brachte.

An dieser Stelle ist vielleicht die Tatsache wichtiger, dass das damalige intellektuelle Leben weniger segmentiert war. So wurden gewöhnlich nicht nur Natur- und Geisteswissenschaften (um den heutigen Sprachgebrauch zu verwenden) in denselben Kreisen und oft von denselben Personen erörtert, sondern die Theologie war auch Teil der aktuellen Diskussion. Ein Teil dessen, was Smith als Professor für Moralische Philosophie in Glasgow lehrte, war die „Natürliche Theologie“. Einer der bedeutendsten Texte für den Unterricht in Natürlicher Theologie im 18. Jahrhundert in Schottland war wiederum Newtons Principia Mathematica. Wenn die „Literati“ der Schottischen Aufklärung auswärts speisten, so waren regelmäßig Theologen unter ihren Gesprächspartnern. Zu den mehr als 100 Mitgliedern der Select Society, Edinburghs exklusivem Dining- und Debattierclub, zu dem Smith, David Hume, Adam Ferguson und die meisten angesehenen schottischen Persönlichkeiten dieser Zeit zählten, gehörten 14 Minister (inklusive Ferguson, der den Lehrstuhl für Moralphilosophie in Edinburgh innehatte, sowie William Robertson, dem Vorsitzenden der Moderates in der schottischen Kirche und Präsident der Universität von Edinburgh). ${ }^{3}$

Infolgedessen waren Smith und seine Zeitgenossen permanent den damaligen aktuellen Diskussionen, Kontroversen und neuen Ideen in der Theologie ausgesetzt, genauso wie Ökonomen im heutigen Universitätsleben mit neuen Theorien der Physik, Biologie oder Bevölkerungswissenschaft in Kontakt kommen. Und genauso, wie Ökonomen heute Ideen aus anderen Forschungsrichtungen aufgreifen - man denke nur an Gravitationsmodelle des Handels oder an den Contagion-Effekt bei Finanzoder Währungskrisen -, könnten die Theoretiker des 18. Jahrhunderts, die die Ökonomik als eigenständige Disziplin entwickelten, durchaus von dem beeinflusst worden sein, was sie von religiösem Denken hörten, lasen und sahen. Um es klar auszudrücken: Ich unterstelle nicht, dass Smith oder einer der anderen „ökonomischen“ Denker seiner Tage bewusst darauf abzielten, religiöse Grundsätze in ihre Gedanken und

2 Einige Autoren vertreten eine andere Auffassung; vgl. z.B. Jerry Evensky (1998).

3 Vgl. Roger L. Emersin (1973). 
Schriften mit einzubeziehen. Vielmehr beeinflussten die theologischen Kontroversen, welchen sie ausgesetzt waren, ihre grundlegende Sicht auf den Menschen und die Welt - ihre „pre-analytic vision“, um Joseph A. Schumpeters (1954) Begriff dafür zu verwenden - und eben jene Sicht ließen sie in ihre Auffassungen einfließen.

\section{Die Smithsche Revolution}

Und es war dieses neue Denken, das uns die Wirtschaftswissenschaft als die akademische Disziplin brachte, die wir heute kennen. Das zentrale Theorem, das der heutigen westlichen Ökonomik zugrunde liegt - nämlich das Konzept, das wir als Adam Smiths „unsichtbare Hand“ kennen (obwohl Smiths Verwendung dieses Ausdrucks, den er lediglich einmal in der Theorie der ethischen Gefüble und ein weiteres Mal im Woblstand der Nationen verwendet, weder innovativ noch besonders spezifisch war ${ }^{4}$ - ist, dass rein eigennutzorientiertes Verhalten von Individuen zu vorteilhaften Ergebnissen nicht nur für die betroffenen Individuen selbst, sondern auch für andere führen kann und unter den richtigen Bedingungen führen wird. Obwohl es bereits Vorläufer gab (am offenkundigsten in den Schriften von Pierre Nicole (1696), dessen Gedanken auch einen klar erkennbaren Ursprung im theologischen Denken hatten - in seinem Fall im Jansenismus resp. bei Augustinus), ${ }^{5}$ war das Konzept, vor allem in der von Smith hervorgebrachten Form, neu und erwies sich als einflussreich.

Zu Beginn des 18. Jahrhunderts sprach ein Teil derjenigen, die sich mit diesen Themen beschäftigten, dem Einzelnen die Fähigkeit zu, erkennen zu können, welche Handlungen und welches Streben ihm nutzen; andere sprachen den Menschen diese Fähigkeit ab. Es fehlte jedoch das Verständnis dafür, dass das Verfolgen des Eigeninteresses, selbst wenn sie dieses korrekt wahrnahmen, allgemein vorteilhafte Ergebnisse hatte. Tatsächlich war „lasterhaft“ das übliche Adjektiv, mit dem eigennutzorientiertes Verhalten charakterisiert wurde.

Der ernsthafte Meinungsumschwung begann mit Bernard Mandevilles Bienenfabel, die erstmals im Jahr 1714 und in überarbeiteter Form im Jahr 1723 veröffentlicht wurde. Bekanntlich vertrat Mandeville die grundlegende Einsicht, dass individueller Eigennutz zu allgemein vorteilhaften Ergebnissen führen könne. Allerdings hat er weder diesen Gedanken noch die Bedingungen, unter welchen dieser Vorteil zum Tragen kommt, vollkommen entfaltet. Wie sein Untertitel deutlich macht („Private Laster, öffentliche Vorteile"), betrachtete er dieses Verhalten folglich weiterhin als „lasterhaft". Aber Mandevilles Fabel führte zu einer umfassenden Diskussion, und vieles von dem, was Hume, Francis Hutcheson und andere über diese Fragen in der Mitte des 18. Jahrhunderts schrieben, war eine Reaktion auf Mandeville.

Smiths Woblstand der Nationen, veröffentlicht im Jahr 1776, entwickelte vollständig die Idee, wonach private Interessen zum Gemeinwohl führen, wie wir sie heute kennen: Individuen nehmen ihr Eigeninteresse zutreffend wahr (in ihrer Rolle als Produzenten, wenngleich nicht unbedingt als Konsumenten); ihr Verlangen, ihren Eigennutz zu verfolgen, ist ein wesentliches Merkmal der menschlichen Natur; ihr entsprechendes

4 Vgl. Emma Rothschild (2001, Kap. 5) für eine Diskussion von Smiths Gebrauch des Ausdrucks und für Verweise auf frühere Untersuchungen.

5 Vgl. Albert O. Hirschmann (1977) für eine knappe Zusammenfassung einiger wichtiger Vorläufer. 
Verhalten führt unter den richtigen Bedingungen zu Resultaten, die in einem umfassenderen Sinn optimal sind; und die wesentliche Bedingung, die diese allgemeinen optimalen Ergebnisse hervorbringt, ist der Wettbewerb auf den Märkten. Es ist nicht überraschend, dass im Woblstand der Nationen die Begriffe „Laster“ und „lasterhaft“ verschwunden sind. Gegen Ende des Jahrhunderts war Smiths Ansicht weit verbreitet und wurde allgemein akzeptiert.

\section{Religiöse Debatte in Adam Smiths Gesellschaft}

Aber was hat das alles mit religiösem Denken zu tun?

Der zentrale Impuls der latitudinarischen Auseinandersetzung innerhalb der Kirche von England, die ihren Höhepunkt etwa ein halbes Jahrhundert vor Mandevilles Bienenfabel fand (Mandeville war Niederländer, lebte aber in London und schrieb auf Englisch), sowie jener Impuls der Auseinandersetzung zwischen den Moderates und den Evangelicals in Schottland während eines Großteils von Smiths Erwachsenenlebens war ein bedeutendes und kontroverses Umdenken, das viele Religionshistoriker als den „Niedergang des Calvinismus" 6 bezeichneten - obgleich es für Fachfremde hilfreich sein mag, es den Niedergang des orthodoxen Calvinismus zu nennen. Drei Schlüsselelemente dieses Übergangs stimmten zumindest bemerkenswert überein mit drei Aspekten des Übergangs von der Ablehnung des „lasterhaften“ Verfolgens des Eigeninteresses (was die Menschen wohl ohnehin nicht als solches empfunden haben) hin zu der Erkenntnis, dass ein solches Verhalten unter den richtigen Rahmenbedingungen zu allgemeinen vorteilhaften Ergebnissen führen würde: (1) Orthodoxe Calvinisten glaubten an die „vollkommene Verdorbenheit“ der Menschen. Ihre Widersacher innerhalb der englischsprachigen protestantischen Welt glaubten größtenteils an das Gute im Menschen (und daran, dass alle Menschen potentiell zur Erlösung auserwählt sind). (2) Orthodoxe Calvinisten glaubten an die Vorbestimmung, wobei menschliche Entscheidungen und menschliches Handeln keinen Einfluss darauf hatten, wer gerettet wird und wer nicht. Ihre Kontrahenten glaubten nicht nur, dass potenziell jeder gerettet werden könne, sondern auch, dass die Entscheidungen und Handlungen des Einzelnen - die schöpferische Tätigkeit des Menschen - eine Rolle in dieser Bestimmung spielten. Wie der latitudinarische Erzbischof von Canterbury, John Tillotson, der infolge der Glorreichen Revolution ernannt wurde, es ausdrückte, können Menschen bei ihrer eigenen Rettung „mitwirken“ und „,von Gott kann nicht verlangt werden, denen zu helfen und beizustehen, die selbst nichts dazu beitragen" ${ }^{7}{ }^{7}$ (3) Orthodoxe Calvinisten glaubten, dass der einzige Grund für die Existenz der Menschheit die Verherrlichung Gottes sei. Ihre Kontrahenten glaubten, dass menschliches Glück auch eine legitime, von Gott vorgesehene Absicht sei.

Obwohl die Beziehung dieser Veränderungen im religiösen Denken zum anschließenden Wandel im ökonomischen Denken wohl kaum als exakt bezeichnet werden kann, gibt es trotzdem einen auffälligen Zusammenhang. Die Überzeugung, dass Männer und Frauen mit einer inhärenten Güte geboren werden, regt eher zu der Vorstellung an, dass sie ihr Eigeninteresse begreifen und von Nutzen für andere sein können -

\footnotetext{
$6 \quad$ Vgl. beispielsweise Daniel Walker Howe (1972).

$7 \quad$ Zitate sind von John Tillotson (1748).
} 
insbesondere dann, wenn das Glück des Menschen ein göttlich zugesichertes Ziel menschlicher Existenz ist, als wenn sie vollkommen verdorben im religiösen/moralischen Sinn sind. Die weitere Überzeugung, dass alle Frauen und Männer grundsätzlich zur Erlösung bestimmt sind - und weiterhin, dass menschliches Handeln Teil dessen ist, was diese Erlösung ermöglicht - legt eher nahe, dass das Handeln der Individuen in ihrem wohlverstandenen Eigeninteresse nicht nur ihr eigenes Leben verbessern kann, sondern auch das ihrer Mitgeschöpfe, als die Überzeugung der Prädestinationslehre, dass nur wenige gerettet werden und dass menschliches Tun keinen Einfluss auf die Rettung hat.

Noch ein weiterer Wandel in den religiösen Vorstellungen, der etwa zur gleichen Zeit, jedoch nicht als Teil der Calvinisten- bzw. Anticalvinisten-Debatte stattfand, betraf die Zukunft des Menschen auf der Erde. Kurz gesagt, während diejenigen, die schließlich als „Prämillenaristen“ bekannt wurden, einen eschalogischen Pessimismus zeigten, indem sie glaubten, dass ausschließlich die Wiederkunft Christi und die sich daraus ergebende Vernichtung der Welt, so wie wir sie kennen, zu grundlegenden Verbesserungen führen könnten, waren die neueren „Postmillenaristen“ der Überzeugung, dass die in der Bibel vorausgesagten tausend Jahre glückseligen Lebens Teil der menschlichen Geschichte sein würden und dass menschliches Handeln seinen Teil zur Realisierung beitragen würde. Diese Überzeugung, dass der „Fortschritt“ in den Lebensbedingungen, herbeigeführt durch menschliches Tun, nicht nur möglich ist, sondern auch dabei hilft, das Millennium zeitlich näher zu bringen, stimmt ebenfalls weit mehr mit der gleichen Ansicht über die vorteilhaften Auswirkungen - für sich selbst sowie für andere - des eigennutzorientierten Handelns der Individuen in der wirtschaftlichen Sphäre überein. (Als der Postmillenarismus im 19. Jahrhundert weiterhin an Stärke gewann, wurden in vielen protestantischen Kreisen herausragende ökonomische/wissenschaftliche Fortschritte wie das Verlegen des ersten transatlantischen Telegraphenkabels als bedeutsam für das Millennium gewürdigt.)

\section{Religiöses Denken und ökonomisches Denken}

Um es erneut deutlich zu machen: Es gibt nur wenige oder gar keine Hinweise darauf, dass Smith oder Mandeville oder irgendein anderer ausschlaggebender Denker dazwischen, außer Hutcheson, irgendeine dieser religiösen Überzeugungen teilte. Der Punkt ist vielmehr, dass sie permanent den Auseinandersetzungen in diesem Verlauf ausgesetzt waren. Darüber hinaus erhöhte die Tatsache, dass diese Überzeugungen weder im England des ersten Viertels noch in Schottland des dritten Viertels des 18. Jahrhunderts vollständig akzeptiert waren, ihre Sichtbarkeit und ihre herausragende Bedeutung vermutlich umso mehr. Die meisten Menschen schenken Ansichten, die von allen akzeptiert werden und die die meisten für selbstverständlich erachten, nur wenig Aufmerksamkeit (obwohl Smith selbstverständlich Moralphilosoph war, und zwar ein tiefgründiger und erkenntnisreicher). Was Aufmerksamkeit auf sich zieht und Auseinandersetzungen auslöst, sind umstrittene Behauptungen, deren Implikationen ein Spannungsfeld erzeugen. Thesen, die der offiziellen Lehre entgegenstehen, ziehen gewöhnlich besondere Aufmerksamkeit auf sich. Die protestantische Theologie unterzog sich damals einer höchst strittigen Reform, die sowohl Mandeville als auch Smith hautnah miterlebten. 
Es wäre auch schwierig zu behaupten, dass eine dieser Bewegungen innerhalb des religiösen Denkens, die weg vom orthodoxen Calvinismus führten, für die nachfolgende Veränderung im ökonomischen Denken unbedingt notwendig gewesen wäre. Smiths Ideen sind wirkmächtig, und sie stehen für sich. Es sollte auch nicht die Behauptung aufgestellt werden, dass alle vier Veränderungen in den religiösen Ansichten zusammen ausreichend dafür gewesen wären. Mandeville und Smith brachten diese Gedanken auf, sie waren aber - zumindest in der damaligen Zeit - nicht unumgänglich. Aber angesichts der offensichtlichen Bezüge zwischen diesen neuen und umstrittenen religiösen Vorstellungen und den zentralen Elementen des geistigen Wandels im 18. Jahrhundert hin zu dem, was dann die Ökonomik wurde, bleibt viel Raum, um gemäß Schumpeter - der Auffassung zu sein, dass diese Veränderungen im theologischen Denken dabei halfen, eine neue Sicht auf die Rolle der Individuen zu eröffnen, die in hohem Maße zur Smithschen Revolution beitrug sowie dazu, deren Akzeptanz zu erhöhen.

Die Verbindung, die ich hier zwischen dem theologischen Wandel weg vom Calvinismus und der ökonomischen Revolution hin zu Smith andeute, weist sowohl Parallelen als auch Gegensätze zu Max Webers (1905) klassischer Hypothese der protestantischen Ethik auf. Wie bei Weber identifiziert sie eine Kausalbeziehung von religiösen Ideen zur Ökonomik. Und wie auch bei Weber überdauerte das, was ursprünglich die Folge eines religiösen Anstoßes war, die Atrophie des eigentlichen religiösen Impulses. Weber wählte Benjamin Franklin (erneut allenfalls ein Deist) als seinen „Idealtypen“ um zu zeigen, dass die Hingabe an "calvinistische“ Tugenden wie Fleiß oder Sparsamkeit auch in einem späteren, säkularisierten Zeitalter andauerte. Im Falle der Smithschen Revolution, in der - um es vorweg zu nehmen - die beteiligten Personen keine religiösen Menschen im eigentlichen Sinn waren, folgt noch ganz selbstverständlich, dass die neuen Denkweisen, die ursprünglich zum Teil durch neue religiöse Vorstellungen belebt worden waren, das Verschwinden dieser religiösen Diskussionen aus der öffentlichen Aufmerksamkeit überleben sollten.

Aber es gibt auch Unterschiede. Bei Weber wird ein Einfluss religiöser Vorstellungen auf das ökonomische Verhalten postuliert; hier hingegen geht es um ökonomische Ansichten. Und noch wichtiger ist die Tatsache, dass die hier von mir vorgebrachte Argumentation hinsichtlich des Gehalts der religiösen Vorstellungen Weber direkt zuwiderläuft. Bekanntlich argumentierte Weber, dass das, was wirtschaftliches Verhalten hauptsächlich antrieb - Verhalten, welches er in protestantischen Ländern und in den überwiegend protestantischen Regionen in seinem eigenen Land zu beobachten glaubte - ein Vermächtnis der Prädestinationslehre gewesen sei. Hier ist das zentrale Moment das Verschwinden des Glaubens in die Prädestination. Insofern ist die Argumentation hier „Weber auf den Kopf gestellt“.

\section{Fazit}

Die Rolle religiöser Ansichten bei der Entstehung der Ökonomik als eigenständige intellektuelle Disziplin, mit einem ihr eigenen Forschungsgebiet und ihr eigenen analytischen Werkzeugen, formte auch die Entwicklung des Fachgebiets weit über den grundlegenden Wandel im 18. Jahrhundert hinaus, der in Smiths Woblstand der Nationen gipfelte. Religiöse Ansichten beeinflussten vor allem in Amerika erkennbar die Art 
und Weise, wie die Smithsche Revolution aufgenommen wurde. Die Autoren der Lehrbücher zur Nationalökonomie, welche die Zeit vor dem Bürgerkrieg dominierten - namentlich John McVickar (1830) an der Columbia University sowie Francis Wayland (1837) an der Brown University und Francis Bowen (1856) an der Harvard University - waren alle entweder geweihte Geistliche oder eng verbunden mit der einen oder anderen protestantischen Glaubensgemeinschaft. ${ }^{8}$ In der Zeit des Bürgerkriegs war die Bewegung, die die Ökonomik zu einem Bemühen um Fortschritt werden ließ (Fortschritt, welcher Wohlfahrtskriterien voraussetzt, ist ein inhärent moralisches Unterfangen), sogar noch bewusster von religiösem Gedankengut beflügelt. Viele der führenden Persönlichkeiten, welche die American Economic Association gründeten, einschließlich John Bates Clark und Richard T. Ely, hatten ihre intellektuellen Wurzeln in der damals neu aufkommenden Social-Gospel-Bewegung. Sogar noch heute sind viele der offenen Fragen, welche die öffentliche Diskussion sowohl über ökonomische Grundsätze als auch über die Wirtschaftspolitik betreffen, ein Widerhall dessen: - Warum vermeiden die meisten Ökonomen Modelle, in denen die Anfangsbedingungen die Endergebnisse determinieren? Warum gibt es ein „angelsächsisches Modell“" der Wirtschaftspolitik? Warum unterstützen so viele Amerikaner leidenschaftlich die Abschaffung der Erbschaftssteuer (,death taxes“), obwohl sie keine Aussichten darauf haben, steuerpflichtiges Vermögen zu erben?

Kritiker beanstanden manchmal, dass der Glaube in freie Märkte nicht nur von Ökonomen, sondern auch von gewöhnlichen Bürgern, ebenfalls eine Art von Religion sei. Es zeigt sich, dass diese Meinung einen wahren Kern hat - zwar nicht auf die Weise, wie die Kritiker meinen, sondern in einem tieferen, mehr historisch fundierten Sinn. Ein besseres Verständnis dieses andauernden Widerhalls, seines Ursprungs und seiner anschließenden Implikationen für die Veränderung des ökonomischen Kontextes über die Zeit würde die Würdigung der Ökonomik als moralische Wissenschaft erhöhen.

\section{Literaturverzeichnis}

Bowen, F. (1856): Principles of Politcal Economy: Applied to the Condition, the Resources and the Institutions of the American People, Boston: Little, Brown.

Davenport, S. (2008): Friends of the Unrighteous Mammon: Northern Christians \& Market Capitalism, 1815-1860. Chicago: University of Chicago Press.

Emerson, R. L. (1973): The Social Compositions of Enlightened Scotland: The Select Society of Edingburgh, 1754-1764, in: Studies on Voltaire and the Eighteenth Century, Vol. 114, 291-330, Oxford: Voltaire Foundation.

Evensky, J. (1998): Adam Smith's Moral Philosophy: The Role of Religion and its Relationship to Philosophy and Ethics in the Evolution of Society, in: History of Political Economy, Vol. 30/No.1, 17-42.

Hirschmann, A. O. (1971): The Passions and the Interests: Political Arguments for Capitalism Before its Triumph, Princeton: Princeton University Press.

Howe, D. W. (1972): The Decline of Calvinism: An Approach to its Study, in: Comparative Studies in Society and History Vol. 14/No.3, 306-327. 
Mandeville, B. (1924 [1723]): The Fable of the Bees: Or Private Vices, in: Kaye, F. B. (Ed.): Publick Benefits, Oxford: Oxford University Press, 1924.

McVickar, J. (1830): Introductory Lecture to a Course on Political Economy, London: J. Miller.

Nicole, P. (1696): Of Charity and Self-Love, in: Moral Essays, London: Samuel Manship.

Rothschild, E. (2001): Economic Sentiments: Adam Smith, Condorcet and the Enlightenment, Cambridge: Harvard University Press.

Schumpeter, J. A. (1954): History of Economics, Schumpeter, E. B. (Ed.), New York: Oxford, University Press. 78-112.

Smith, A. (1776): An Inquiry into the Nature and Causes of the Wealth of Nations, Campbell, R. H./Skinner, A. S. (Eds.), Oxford: Oxford University Press, 1976.

Smith, A. (1974 [1759]): The Theory of Moral Sentiments, übers. durch H. C. Recktenwald, München: Beck.

Tillotson, J. (1748): Of The Nature of Regeneration and its Necessity, in order to Justification and Salvation, In the works of the most Reverend Dr. John Tillotson, Late Archbishop of Canterbury, Vol. 5, sermon 106, Edinburgh: G. Hamilton.

Wayland, F. (1837): The Elements of Political Economy, New York: Leavitt, Lord.

Weber, M. (1930 [1905]): The Protestant Ethics and the Spirit of Capitalism, transl. by T. Parsons, London: George Allen \& Unwin. 